\title{
Dynamic Programming-based Optimization of Electric Vehicle Fleet Charging
}

\author{
Branimir Škugor and Joško Deur \\ University of Zagreb, Faculty of Mechanical Engineering and Naval Architecture \\ Zagreb, Croatia \\ branimir.skugor@fsb.hr, josko.deur@fss.hr
}

\begin{abstract}
The paper deals with charging optimization for delivery electric vehicle fleets based on dynamic programming method. Charging of each individual vehicle within the fleet is optimized separately, thus providing globally optimal solution on the vehicle level. By posing an upper constraint on the grid power used for charging, the individual charging optimizations are coupled together on the fleet level in a suboptimal way. Consecutive optimizations for each vehicle within the fleet are conducted for different orders of individual vehicle optimizations. In this way the sensitivity of optimization results with respect to ordering of charging optimizations can be analysed and a solution closer to global optimum can be found. The obtained optimization results are used for the purpose of validation of previously developed aggregate battery models and corresponding heuristic method dealing with distribution of optimal aggregate power over individual vehicles.
\end{abstract}

Keywords-electric vehicle; fleet; modeling, charging; optimization; dynamic programming

\section{INTRODUCTION}

Electric vehicles can provide several distinct benefits compared to their conventional counterparts such as reduced emissions of air pollutants and reduced cost of energy consumption [1]. The energy cost reduction becomes more emphasized when using smart charging algorithms, which are typically based on various optimization methods. This is particularly beneficial for isolated vehicle fleets (e.g. delivery vehicle fleets), where vehicle driving patterns are repeatable or highly predictable [2], as well as for deregulated electricity markets with highly variable energy cost. Apart from the energy cost reduction, electric vehicles can provide additional cost benefits by offering ancillary services to the grid, e.g. regulation services [3]. In order to enable this possibility, an aggregator which represents smart interface between electric vehicle fleet and the grid should be introduced [4, 5].

In literature there are several approaches to investigate impact of electric vehicles charging on the electrical grid and cost benefits of replacing conventional vehicles with electric ones. They can be categorized in two major categories: (i) individual battery-based and (ii) aggregate battery-based approaches. Individual battery-based approach deals with modelling and charging optimization of a single electric vehicle represented by a single battery $[3,6]$. In $[3,6]$ the optimization time horizon of one day is assumed with the electricity price time distribution known in advance, and the dynamic programing (DP) method is applied for charging optimization to obtain the globally optimal results which are compared with the fast charging results. The aggregate battery modelling approach is generally used when a large number of electric vehicles is subject of the analysis, e.g. for energy planning studies of whole cities or states [7, 8, 9, 10]. In [4] an aggregator of electric vehicles is presumed, but the optimal charging problem is formulated on the individual vehicle level and solved by using the DP algorithm. It is assumed that the charging schedule optimality of a single vehicle will lead to an optimality on the aggregate level. However, this holds only if the grid constraints are neglected, i.e. in the presence of the grid constraints the solution will be sub-optimal. In [5] and [9] charging optimizations of aggregate battery models are performed by using quadratic programming and dynamic programming, respectively. In both approaches, the optimal aggregate power profiles are distributed to individual batteries using proper distribution algorithms.

This paper deals with modelling and DP-based vehiclelevel charging optimization of a delivery electric vehicle fleet. Each vehicle within a fleet is represented by a single battery model. Since the number of vehicles is relatively small, charging optimizations of all vehicles are performed separately (in a consecutive manner) and coupled together through the maximum grid power constraint. Since the order of individual vehicle optimizations can influence the final fleet charging results, the optimizations are repeated for different orders and the corresponding sensitivity of the final results is analysed. The optimization results are used as a benchmark to validate the aggregate battery models developed in the previous authors' publications and used therein for aggregate-level charging optimization $[9,10]$.

\section{BATTERY MODELS}

\section{A. Individual battery model}

Battery of each vehicle is modelled as an energy storage. The energy level of each battery is expressed in terms of normalized state-of-charge $(\mathrm{SoC})$ variable (actually, state of energy variable), and the corresponding battery state equation is defined as 


$$
S o C(k+1)=S o C(k)+\eta_{c h} \frac{\left(P_{c}(k)+P_{r e g}(k)\right) \Delta T}{E_{\max }}-\frac{P_{d e m}(k) \Delta T}{\eta_{d c h} E_{\max }},
$$

where $P_{c}$ and $P_{\text {reg }}$ are the battery charging power values related to charging from the grid and regenerative braking, respectively; $P_{d e m}$ is the vehicle power demand; $\eta_{c h}$ and $\eta_{d c h}$ are charging and discharging efficiencies, respectively; $E_{\max }$ is the battery energy capacity; and $k$ denotes the discrete time step and takes values between 1 and $N_{t}$, where the time length of each discrete time step is denoted with $\Delta T$. The following constraints related to the lower and upper limits of $S o C$, and the lower and upper limit of charging power are imposed to the individual battery model (i.e. to charging optimization).

$$
\begin{aligned}
& 0 \leq S o C_{\text {min }} \leq \operatorname{SoC}(k) \leq S o C_{\text {max }} \leq 1 . \\
& 0 \leq P_{c}(k) \leq n_{d c}(k) P_{c, \text { lim }} .
\end{aligned}
$$

The constant $P_{c, \text { lim }}$ relates to the maximum charging power of individual batteries, while $n_{d c}(k)$ denotes the time share of parked (i.e. ready-to-charge) individual vehicle within each discrete time step $k$. According to Eqs. (1) and (3), the individual battery model requires the following input time distributions: (i) power demand distribution $P_{d e m}(k)$, (ii) regenerative braking power distribution $P_{\text {reg }}(k)$, and (iii) parking time share distribution $n_{d c}(k)$.

\section{B. Aggregate battery models}

In the aggregate battery modelling approach, batteries of all vehicles within a fleet are modelled as a single, so called aggregate battery with a single $S o C$ state variable and a single charging power input. Two different aggregate battery models are considered: the basic one adopted from [7] and somewhat modified (AGGR1) and the novel one proposed in [10] (AGGR2).

The basic aggregate battery model (AGGR1) is described by the following state equation (cf. (1)):

$$
\begin{aligned}
\operatorname{SoC}_{a g g}(k+1)= & \operatorname{SoC}_{\text {agg }}(k) \\
& +\eta_{c h} \frac{\left(P_{c, a g g}(k)+P_{\text {reg }, a g g}(k)\right) \Delta T}{E_{\text {max }, a g g}}-\frac{P_{d e m, a g g}(k) \Delta T}{\eta_{d c h} E_{\text {max }, a g g}},
\end{aligned}
$$

where $S o C_{a g g}$ and $P_{c, a g g}$ correspond to the aggregate battery state variable and charging power, respectively. The constant $E_{\text {max agg }}$ represents the total energy capacity of the aggregate battery, which is simply calculated as the sum of individual battery capacities of all vehicles within the fleet. The accompanying $\mathrm{SoC}$ and charging power constraints are given by (cf. (2) and (3)):

$$
\begin{aligned}
& 0 \leq P_{c, \text { agg }}(k) \leq n_{d c, a g g}(k) P_{c, \lim }, \\
& 0 \leq S o C_{a g g, \min } \leq S o C_{a g g}(k) \leq S o C_{a g g, \max } \leq 1,
\end{aligned}
$$

where $n_{d c, \text { agg }}(k)$ corresponds to the distribution of total number of parked vehicles, i.e. those connected to the grid. According to Eqs. (4) and (5), the basic battery model requires the following input time distributions: (i) transport power demand
$P_{\text {dem,agg }}(k)$, (ii) transport regenerative braking power $P_{\text {reg,agg }}(k)$, and (iii) total number of parked/connected vehicles $n_{d c, a g g}(k)$.

The main drawback of the basic model relates to the assumption that the total aggregate battery capacity $E_{\text {max,agg }}$ is constant and thus available at all times. This is found unrealistic since a portion of vehicles will be on road within each hour and thus not connected to the grid. In order to improve the modelling accuracy, a novel model of aggregate battery has been proposed in [10] and outlined below.

The novel model (AGGR2) state equation is:

$$
\begin{array}{r}
S o C_{a g g}(k+1)=S o C_{a g g}(k)+S o C_{i n, a v g}(k) \frac{\Delta n_{i n, d c}(k)}{N_{v}}- \\
S o C_{\text {out }, a v g} \frac{\Delta n_{\text {out }, d c}(k)}{N_{v}}+\eta_{c h} \frac{P_{c, a g g}(k) \Delta T}{E_{\text {max }, a g g}},
\end{array}
$$

where $\Delta n_{i n, d c}$ and $S o C_{i n, a v g}$ correspond to the number of vehicles connecting to the grid within each time step $k$ and their average $S o C$, respectively; and $\Delta n_{\text {out,dc }}$ and $S o C_{\text {out avg }}$ correspond to the number of vehicles disconnecting from the grid and their average $S o C$, respectively. Here, $S o C_{\text {out avg }}$ may be regarded as an auxiliary control variable, and it is set to 1 in this paper, assuming that the departing vehicles have full batteries. The constant $N_{v}$ relates to the total (maximum) number of vehicles which can be connected to the grid. In the novel model (7), the battery charging is realized through the charging power $P_{c, \text { agg }}$ as in the case of the basic model (4), while the battery discharging is reflected through the average $S o C$ of vehicles connecting to the grid (Eq. (7)). The upper charging power limit (5) remains unchanged, while the $\mathrm{SoC}$ constraint upper limit is made variable in dependence of the number of vehicles connected to the grid:

$$
0 \leq S o C_{a g g, \min } \leq S o C_{a g g}(k) \leq S o C_{a g g, \max }(k)=\frac{n_{d c, a g g}(k)}{N_{v}} \leq 1 .
$$

According to Eqs. (5), (7), and (8), the novel battery model requires the following input time distributions: (i) number of vehicles connecting to the grid $\Delta n_{i n, d c}$, (ii) number of vehicles disconnecting from the grid $\Delta n_{o u t, d c}$, (iii) average $S o C$ of vehicles connecting to the grid $S o C_{i n, a v g}$, and (iv) total number of vehicles connected to the grid $n_{d c, a g g}(k)$.

The aggregate battery novel model has been proven to provide more accurate description of the electric vehicle fleet compared to the basic model (see [10] for comparative validation results). However, it should be noted that the novel model is more demanding in terms of model parameterization.

\section{PARAMETERIZATION OF BATTERY MODELS}

\section{A. Collection of delivery vehicle fleet data}

In order to parameterize the above battery models and verify different charging optimization methods, driving cycle data of a delivery vehicle fleet of a leading regional retail company have been recorded. A set of ten mid-size delivery 
vehicles was chosen as a good representative of the retail company fleet. The driving cycles were being collected continuously ( 24 hour a day) during a three-month period by using the existing GPS/GPRS equipment modified to run on the sampling time basis of one second [2]. The main recorded signals include the vehicle velocity (with the resolution of 0.1 $\mathrm{km} / \mathrm{h}$ ), the absolute vehicle position, and the cumulative fuel consumption, all acquired with the sampling time of $1 \mathrm{sec}$ (see Fig. 1).

The delivery vehicle mission is to load cargo in the main distribution centre and to distribute it to one or more sale centres. The overall set of recorded data are separated into driving cycles, where each driving cycle corresponds to a single mission starting with the vehicle departure from the distribution centre and ending with its return (Fig. 1b). The hypothetical electric delivery vehicle fleet is assumed to be charged in the distribution centre, and the vehicles located in the distribution centre are assumed to be connected to the grid. According to these assumptions, the time distributions required by the battery models are extracted from the recorded data on the one hour discretization time basis $(\Delta T=1 \mathrm{~h})[2,10]$ and described in the next subsections.

\section{B. Distributions related to number of grid-connected vehicles}

Fig. 2a shows the distribution of parking time share of one vehicle within each hour during one week (value of one corresponds to the full parking time within one hour). This distribution is used as an input distribution of the individual battery model (1)-(3). Time distributions shown in Figs. 2b-2d are related to the number of vehicles parked in the distribution centre, and the number of vehicles arriving to and departing from the distribution centre, respectively. These distributions are used as input distributions of the aggregate battery models described in the previous section ([9, 10], see Eqs. (5), (7), and (8)). Fig. 2 indicates that an increased vehicles activity is
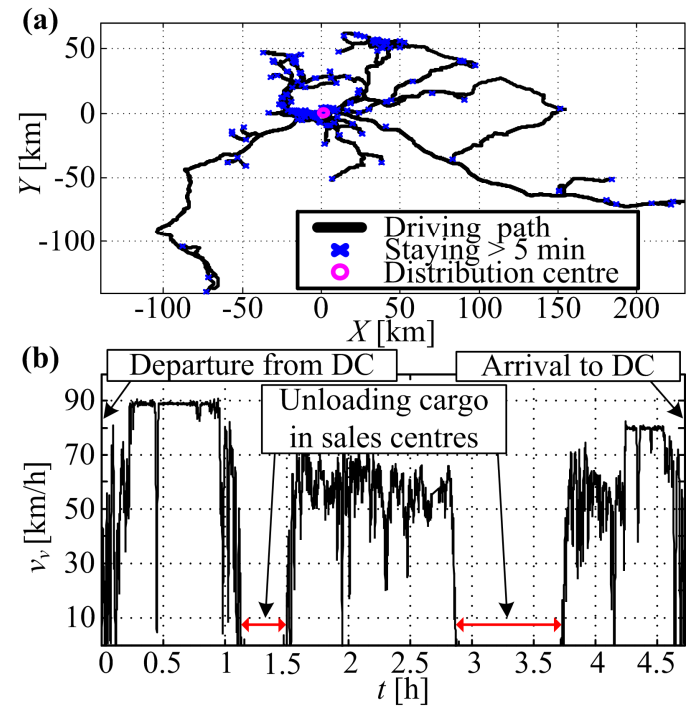

Fig. 1. Recorded driving paths of a vehicle during threemonth period (a) and example of recorded driving cycle (b).

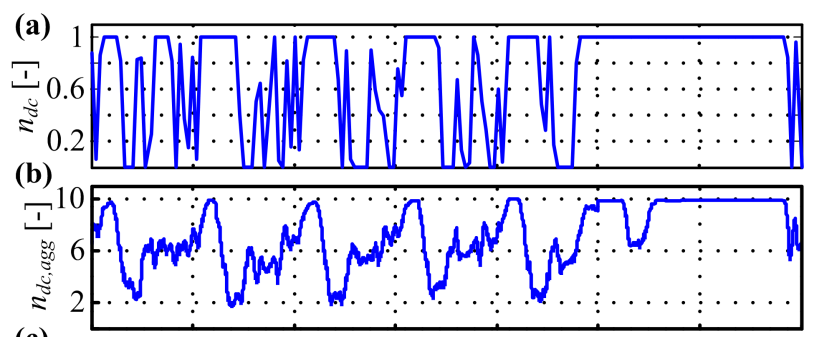

(c)
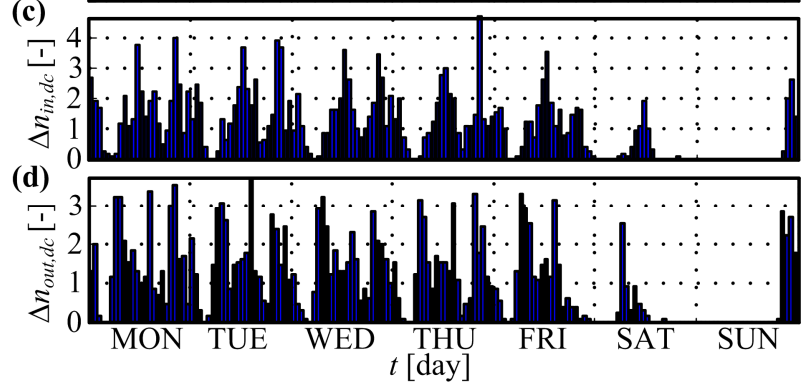

Fig. 2. Weakly time distributions related to number of grid-connected vehicles.

emphasized during working days (Monday-Friday) with a highly repeating pattern, while the vehicles are mostly parked in the distribution centre during the weekend days. On the daily time basis, the vehicle activity is emphasized in the morning hours, while it becomes reduced in the early morning and the late evening hours [2]. These results could be preliminary indicators of charging scheduling of a hypothetic electric vehicle fleet.

\section{Transport demand-related distributions}

In order to obtain the transport demand-related distributions, an extended range electric vehicle (EREV) of Chevy Volt type [11] is modelled and simulated over a set of recorded driving cycles [10]. Even though the considered vehicle fleet is consisted of delivery trucks, the passenger vehicle model can be used for the purpose of proposed battery models validation and corresponding charging approaches verification. However, the more accurate quantitative predictions related to the electricity cost of fleet charging can be obtained by using an electric vehicle truck model of similar powertrain and loading characteristics as the considered conventional truck (see e.g. the e-truck model from [12]).

The EREV powertrain schematic is shown in Fig. 3a. It includes an internal combustion engine (ICE), two electric machines $\left(\mathrm{M} / \mathrm{G}_{1,2}\right)$, an electrochemical battery, three clutches (F1, F2, F3), and a planetary gear as a power-split device. Depending on the clutch state, the vehicle can operate in four distinctive operating modes [11]: single-motor and two-motor electric vehicle modes (EV, TMEV), and series and seriesparallel hybrid electric vehicle modes (SHEV, SPHEV). Fig. $3 \mathrm{~b}$ shows the energy control-oriented backward-looking model of the EREV, where the battery $S o C$ is the only state variable.

The EREV Chevy Volt typically operates as a pure electric vehicle in the charge depleting (CD) regime until its battery $S o C$ reaches predetermined low value (here 0.3 ), after which it switches to the hybrid-like charge sustaining regime (CS) in 


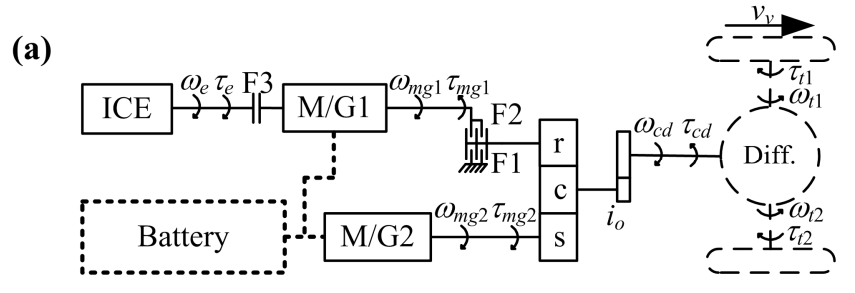

(b)

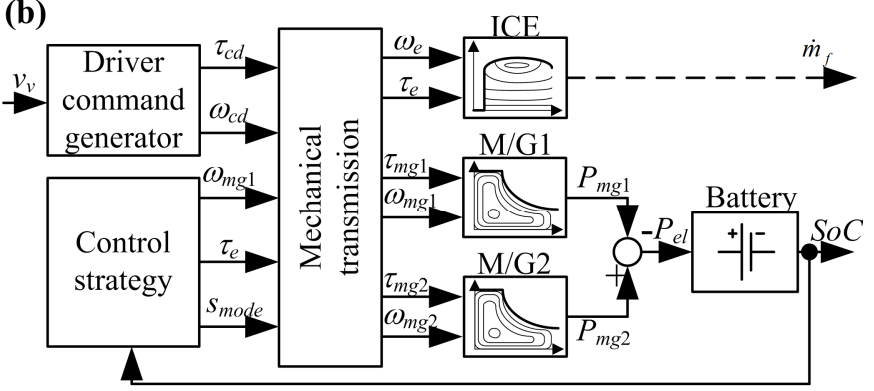

Fig. 3. Schematic of EREV powertrain (a), and EREV

backward-looking model including driver command generator and control strategy (b).

order to prevent deep discharging of the battery. In the CD mode, only electric modes (EV, TMEV) are enabled, and the particular operating mode and corresponding powertrain operating point are chosen with the aim to maximize the electric powertrain efficiency. In the CS mode (SHEV and SPHEV are also enabled), the operating mode and the powertrain operating point are chosen according to predetermined regions of optimal operating modes in the output torque-vehicle speed map and an instantaneous optimisation-based control strategy, respectively. Details on the EREV modelling and feedback control strategy design are given in $[13,14]$. The original vehicle battery model from [14] is reformulated into the energy-based model [10] in order to make it more compatible with the energy-based fleet battery models presented in Section II.

Fig. 4a shows the time distribution of transport power demand for the case of individual vehicle (the same vehicle as considered in Fig. 2a). Naturally, this road activity-related distribution is inverted when compared to parking time share distribution in Fig. 2a. Figs. $4 \mathrm{~b}$ and $4 \mathrm{c}$ show the aggregate transport power demand distribution of the ten vehicle fleet and the distribution of average $\mathrm{SoC}$ of arriving vehicles, which are required by the basic and novel aggregate battery models, respectively. The results in Fig. 4 confirm the trends of reduced vehicles activity over the weekend and emphasized activity over the working days, particularly in the morning hours (cf. Fig. 2).

\section{ELECTRIC VEHICLE FLEET CHARGING OPTIMIZATION}

\section{A. Dynamic programming-based charging optimization of individual vehicle}

The discrete-time cost function to be minimized is formulated as

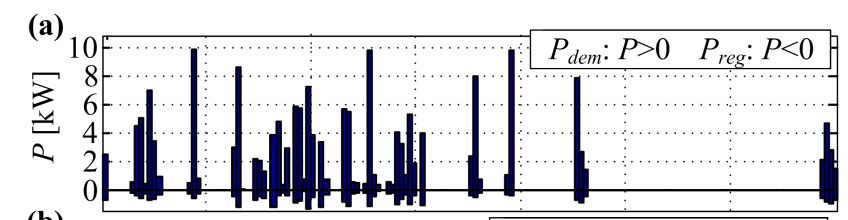

(b)
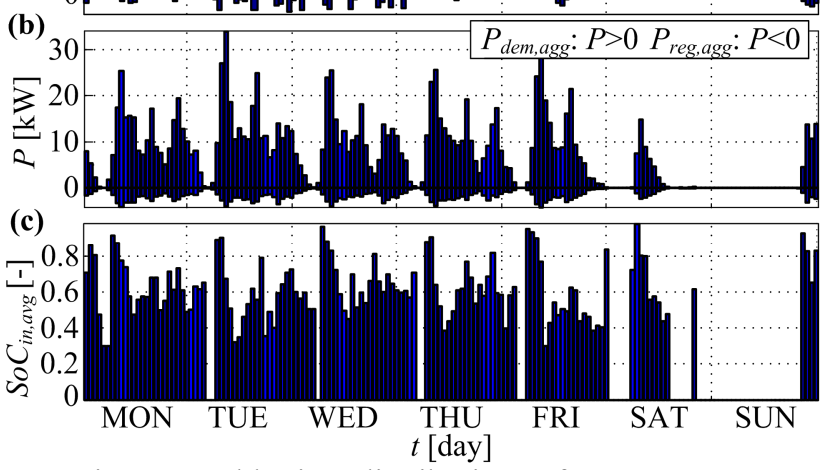

Fig. 4. Weekly time distributions of transport power demand for an individual vehicle (a) and the fleet of 10 vehicles (b), and of average $\mathrm{SoC}$ of arriving vehicles (c).

$J=\sum_{k=1}^{N_{t}} F\left(P_{c}(k), k\right)$,

with the sub-integral term defined as

$$
F\left(P_{c}(k), k\right)=C_{e l}(k) \frac{P_{c}(k) \Delta T}{1000}+L(k), k=1,2, \ldots, N_{t},
$$

where $C_{e l}(k)$ is the electrical energy price per $1 \mathrm{kWh}$ and $L(k)$ is a function that penalizes violations of charging power $P_{c^{-}}$and SoC-related constraints:

$$
L(k)=L_{\text {soc1 }}(k)+L_{\text {soc } 2}(k)+L_{P 1}(k)+L_{P 2}(k)+L_{P_{\text {cum }}}(k) .
$$

Here, the term $L_{s o c 1}$ relates to the lower and upper $S o C$ constraints:

$L_{s o c 1}(k)=\left\{\begin{array}{l}K_{g 1}, \text { for } S o C(k+1)<S o C_{\min } \\ K_{g 2}, \text { for } S o C(k+1)>S o C_{\max } \\ 0, \quad \text { otherwise }\end{array}\right.$.

The term $L_{P 1}$ prevents vehicle charging $\left(P_{c}(k)>0\right)$ in the case when vehicle is not connected to the grid $\left(n_{d c}(k)=0\right)$ :

$L_{P 1}(k)=\left\{\begin{array}{ll}K_{g 3}, & \text { for } n_{d c}(k)=0 \wedge P_{c}(k)>0 \\ 0, & \text { otherwise }\end{array}\right.$.

The penalty function $L_{s o c 2}$ is defined as

$L_{s o c 2}(k)=\left\{\begin{array}{l}K_{g 4}+K_{g 5}\left(1-S o C_{\bmod }(k+1)\right), \text { for } n_{d c, o}(k)=1 \wedge S o C_{\bmod }(k+1)<1 \\ 0, \quad \text { otherwise }\end{array}\right.$

, where $S o C_{\text {mod }}(k+1)$ is obtained as $S o C_{\text {mod }}(k+1)=S o C(k)+$ $\eta_{c h} P_{c}(k) \Delta T / E_{\max }$, and represents the $S o C$ value in the $(k+1)^{\text {th }}$ discrete time step without taking into account vehicle driving power demand from the $k^{\text {th }}$ step (see (1)). By imposing the penalty function (14), the value of $S o C_{\text {mod }}(k+1)$ is forced to be one if vehicle is disconnecting from the grid within the $k^{\text {th }}$ 
discrete time step $\left(n_{d c, o}(k)=1\right)$. This means that a vehicle should be fully charged until instant of disconnecting from the grid.

Another charging power-related penalty function is

$L_{P 2}(k)=\left\{\begin{array}{l}K_{g 6}+K_{g 7}\left(\frac{P_{c}(k)-n_{d c}(k) P_{c, \mathrm{lim}}}{P_{c, \mathrm{lim}}}\right), \text { for } P_{c}(k)>n_{d c}(k) P_{c, \mathrm{lim}}, \\ 0, \text { otherwise }\end{array}\right.$

where $P_{c, \text { lim }}(k)$ is the upper limit of individual battery charging power to be satisfied.

Finally, violation of the total grid charging power limit $\left(P_{\text {cum,lim }}\right)$ is penalized as

$$
L_{P_{\text {cum }}}(k)=\left\{\begin{array}{l}
K_{g 8}+K_{g 9}\left(\frac{P_{c}(k)-P_{\text {cum } \mathrm{lim}}(k)}{P_{\text {cum } \mathrm{lim}}(k)}\right), \text { for } P_{c}(k)>P_{\text {cum } \mathrm{lim}}(k) \\
0, \quad \text { otherwise }
\end{array}\right.
$$

where $P_{\text {cum lim }}$ is made variable in order to couple charging optimizations of different individual vehicles calculated successively through the common constraint on the maximum grid power $P_{\text {cmax, grid }}$ (the details in the subsection B).

The penalty terms (12) and (13) have priorities over the terms (14)-(16), because the $S o C$ limits and the disconnected vehicle charging inability condition represent physical constraints that need to be satisfied. Correspondingly, the weighting factors $K_{g i}(i=1,2,3)$ need to be tuned to values that are high enough to strictly satisfy the inequality constraints related to (12) and (13). On the other hand, the constraints penalized by terms (14)-(16) are implemented as soft constraints, where the constraint violation is penalized in proportion to its magnitude.

In summary, the aim of dynamic programming (DP) optimization is to minimize the cost of individual battery charging energy (see Eq. (10)):

$C_{b a t, \text { ind }}=\sum_{k=1}^{N_{t}} C_{e l}(k) \frac{P_{c}(k) \Delta T}{1000}$,

while satisfying various battery and charging constraints. The control variable which is optimized is the battery charging power $P_{c}(k), k=1,2, \ldots, N_{t}$.

An important aspect of DP optimization is possibility of prescribing the final value of state variable $\left(S o C_{\text {end }}\right)$ by extending the sub-integral function (9) by the following additive penalty term

$$
J_{f}=K_{f}\left|\operatorname{SoC}\left(N_{t}+1\right)-\operatorname{SoC}_{\text {end }}\right| \text {, }
$$

where $K_{f}$ is the weighting factor to be tuned to satisfy the final condition. From the standpoint of charging process sustainability, it is a common approach to set the final battery $\mathrm{SoC}$ equal to the initial $\mathrm{SoC}$.

$\mathrm{SoC}_{\text {end }}=\mathrm{SoC}_{\text {init }}=\operatorname{SoC}(1)$.
The above optimal control problem is solved by using the DP optimization method, which provides globally optimal results for the given resolution of state and control variables [9, 15]. The optimization procedure consists of two basic steps: (a) backward-in-time optimization of charging power for each discrete value of $S o C$ variable in order to minimize cumulative cost function (9), (b) forward-in-time reconstruction of the optimal control variable solution starting from the known initial condition [9]. The DP algorithm solves the optimal control problem in the discrete domain of time, state and control variables, and consequently finite number of discrete values of these variables should be predefined (the constants $N_{t}, N_{s o c}$, and $\left.N_{\text {control }}\right)$. The optimization horizon $N_{t}$ is set to 2184 , which corresponds to 2184 hours over three-month period (91 days). This is because the battery models input data (Figs. 2 and 4) are recorded for the three month period. The parameters of DP optimization formulation are listed in Appendix.

\section{B. Charging optimization of electric vehicle fleet}

The total power drawn from the grid when charging a fleet of $N_{v}$ vehicles is given by

$$
P_{c, \text { cum }}(k)=\sum_{i=1}^{N_{v}} P_{c, i}(k), k=1,2, \ldots, N_{t},
$$

where $P_{c, i}(k)$ is the charging power of $i^{\text {th }}$ vehicle in the $k^{\text {th }}$ discrete time step. The cost of battery charging energy for all vehicles within the fleet is

$C_{\text {bat }}=\sum_{i=1}^{N_{v}} C_{b a t, i n d, i}$,

where $C_{b a t, i n d, i}$ is the cost of battery charging energy of $i^{\text {th }}$ vehicle calculated according to Eq. (17). Charging optimizations of all vehicles within the fleet are performed successively, and the upper (cumulative) limit of charging power for $(i+1)^{\text {th }}$ vehicle is determined from the previously optimized charging power for the $i^{\text {th }}$ vehicle:

$$
P_{\text {cum }, \lim , i+1}(k)=P_{\text {cum }, \lim , i}(k)-P_{c, i}(k), i=1,2, \ldots, N_{v} .
$$

The iterative process (22) is initialized as

$P_{\text {cum }, \text { lim }, 1}(k)=P_{\text {cmax }, \text { grid }}$ for $k=1,2, \ldots, N_{t}$,

which means that vehicles, which are on the top of optimization order list, have more optimization freedom due to the widest charging power limit. This will cause the optimization results to be dependent on the order list, and would, thus, be suboptimal. The issue could be avoided if charging optimizations of all vehicles are performed simultaneously, but this would make the problem excessively computationally expensive, particularly for a large number of vehicles, $N_{v}$. The duration of simultaneous optimization is estimated to last $N_{\text {soc }}^{N v-1} \cdot N_{\text {control }}{ }^{N v-1} \cdot T_{\text {optim,ind, }}$, where $T_{\text {optim,ind }}$ denotes duration of individual vehicle charging optimization, while successive optimization is estimated to last $N_{v} \cdot T_{\text {optim,ind }}$. This is the reason why successive, denoted as DP-IND, and not simultaneous, charging optimization of individual vehicles within the fleet is applied. In order to verify the sensitivity of optimization solution with respect to optimization order, 
several full optimization sequences have been carried out for randomly permuted optimization order lists, and the one that results in the minimum cost function (21) is selected as the 'optimal' one.

\section{Charging optimization of aggregate battery models}

Charging of the aggregate battery models outlined in Section II.B is also optimized using the DP optimization algorithm [9]. The aim is again to minimize charging cost of electric vehicle fleet for the same optimization time horizon $N_{t}$ $=2184$ as in the case of DP-IND optimization. The charging optimization of the basic and novel aggregate battery models from [10] is denoted as DP-AGGR1 and DP-AGGR2, respectively.

The optimal aggregate charging power for the whole fleet is distributed over individual vehicles using a heuristic distribution method proposed in [10], with the results denoted here by symbols DISTR1 and DISTR2 for the AGGR1 and AGGR2 approaches, respectively.

\section{OPTIMIZATION RESULTS}

The successive DP charging optimization for the individual vehicles is carried out for different upper constraint values on the grid power and the Price 1 model of electricity price shown in Fig. 5. . The optimization results shown in Fig. 6 include: the total charging cost $\left(C_{b a t}\right)$, a correlation coefficient $(K)$ of the aggregate charging profiles $\left(P_{c, \text { cum }}(k)\right.$ from Eq. (20)) obtained in the cases when no upper limit (NC) and when the upper limit (C) on the grid power exists, the number of discrete time steps (hours) for which the grid upper limit is violated $(N)$. The correlation coefficient $K$ is used as the measure of similarity and assumes the value of 1 for the maximum correlation, i.e. for equal input signals, and 0 for the case when no correlation between input signals exists [10].

The results in Fig. 6 point out that the impact of the upper limit $P_{c \text { max }, \text { grid }}$ on the optimization results is negligible when $P_{c \text { max grid }}$ takes values from 30 to $50 \mathrm{~kW}$, while it significantly affects the results when $P_{c \text { max }, \text { grid }}$ is less or equal to $20 \mathrm{~kW}$.

Also, the successive DP charging optimization for the individual vehicles is carried out for the case when there is no upper constraint on the grid power $\left(P_{c \max , \text { grid }}=\infty\right)$, and for

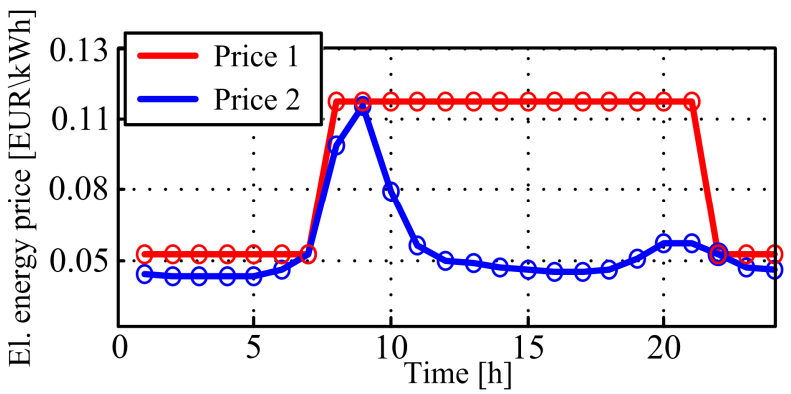

Fig. 5. Two daily electricity price distributions (two-tariff Price 1 model was taken from http://www.hep.hr/, and Price 2 model was adopted from http://www.nordpoolspot.com/).

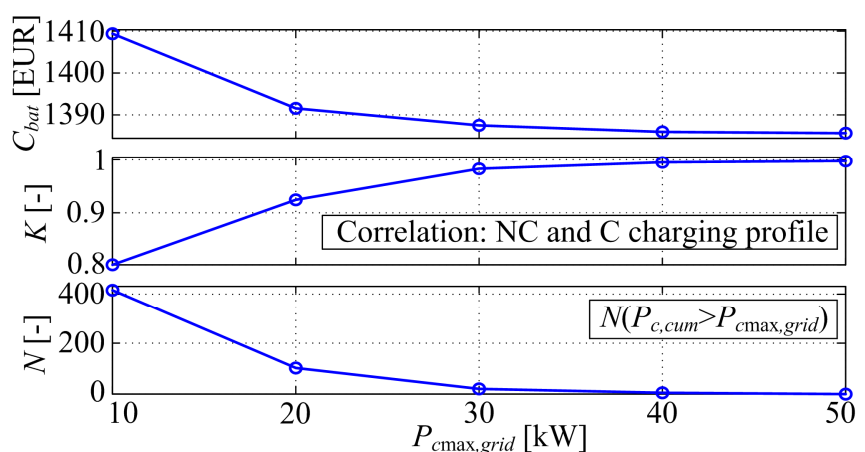

Fig. 6. Dependences of charging cost and grid power violation indices on the upper limit constraint of the grid power (Price 1 model from Fig. 5 is used).

different vehicle charging optimizations ordering lists and the cases of 10 and $20 \mathrm{~kW}$ of upper limit on the grid power. For this analysis two electricity price models from Fig. 5 are considered. The minimized charging cost results, shown in the Fig. 7, indicate that the charging cost increases as the upper limit $P_{\text {cmax, grid }}$ decreases. Also, the charging costs for different optimization ordering lists are densely grouped, which implies that the charging ordering does not have a significant impact on the charging cost, particularly in the case of using Price 1 model (see $C_{b a t, s t d 1}$ Table I). In the case of using Price 2 model, the impact of optimization ordering is negligible in the case $P_{c m a x, \text { grid }}=20 \mathrm{~kW}$, while this is more emphasized for $P_{c m a x, \text { grid }}=$ $10 \mathrm{~kW}$ (see $C_{b a t, s t d 2}$ in Table I).

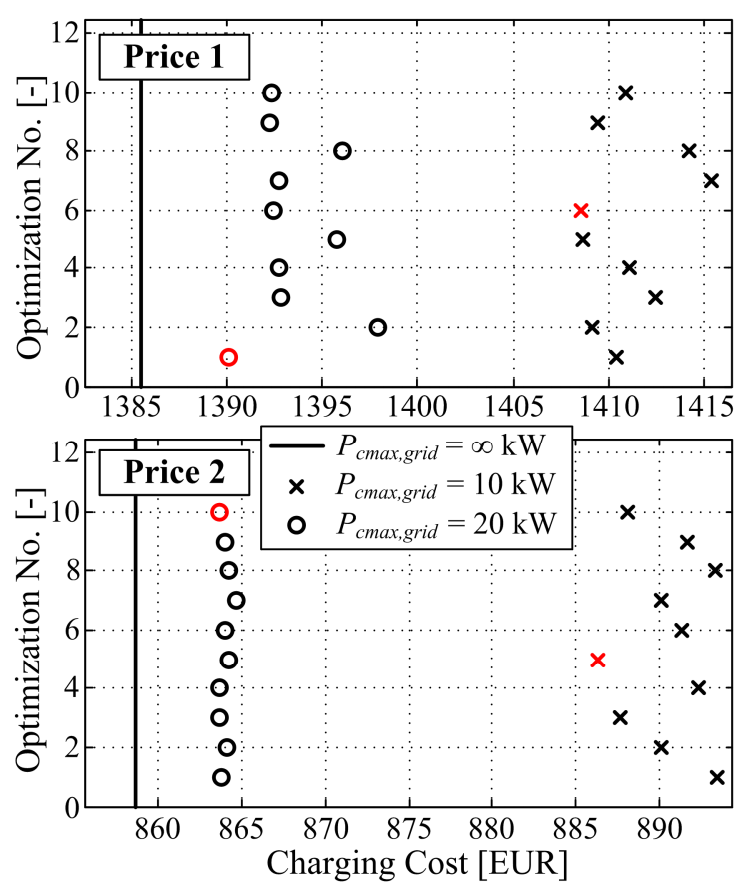

Fig. 7. Optimal charging energy cost for different charging optimization ordering (red circles and red crosses denote optimal charging costs found). 
TABLE I. MINIMUM AND MAXIMUM CHARGING COSTS AND STANDARD DEVIATIONS FOR TEN DIFFERENT OPTIMIZATION ORDERS AND TWO PRICE MODELS FROM FIG. 5

\begin{tabular}{|c|c|c|c|c|c|c|}
\hline \multirow{2}{*}{ Method } & \multicolumn{3}{|c|}{$P_{c m a x, \text { grid }}=10 \mathrm{~kW}$} & \multicolumn{3}{|c|}{$P_{c m a x, \text { grid }}=20 \mathrm{~kW}$} \\
\hline & $C_{b a t, \min 1}$ & $C_{b a t, \max 1}$ & $C_{b a t, s t d 1}$ & $C_{b a t \min 1}$ & $C_{b a t, \max 1}$ & $C_{b a t, \text { std1 }}$ \\
\hline DP & 1408.5 & 1415.4 & 2.34 & 1390.1 & 1397.9 & 2.33 \\
\hline & $C_{b a t, \mathrm{n}}$ & $C_{b a t,}$ & $C_{b a t}$ & $C_{b a}$ & $C_{b a}$ & $C_{b a t, \text { std2 }}$ \\
\hline DP-IND & 886.4 & 893.5 & 2.45 & 863.6 & 864.6 & 0.31 \\
\hline
\end{tabular}

Table II shows the comparison of charging costs for the case of successive DP charging optimization of individual vehicles (DP-IND) and DP charging optimization of aggregate battery models (DP-AGGR1, DP-AGGR2), as well as the charging costs of the corresponding distributed models (DISTR1, DISTR2). The upper limit on the grid power is set to $20 \mathrm{~kW}$. The DP-IND results correspond to the ones with minimum charging costs from the sets of 10 optimizations of different ordering lists (see red circles in Fig. 7). For the twotariff Price model 1 the charging costs of aggregate battery models (DP-AGGR1, DP-AGGR2) are lower than in the case when charging optimization of individual vehicles is applied (DP-IND). In other words, the aggregate battery models underestimate the charging cost (i.e. provide overoptimistic predictions), which is due to inherent inaccuracies of their lumped-parameter formulation. The underestimation of charging cost is particularly emphasized for the DP-AGGR1 approach (20\% compared to only $4 \%$ for DP-AGGR2 approach). This implies that the results obtained by using the novel aggregate battery model [10] are more realistic when compared to the basic model [7].

As a further confirmation AGGR2 model accuracy, the correlation coefficients $\left(K_{1,2}\right)$ are listed in Table II, as the measure of similarity [10] between the constrained DP-IND charging profile, which is considered as optimal result, with charging profiles obtained by using other battery models and charging methods. The correlation is higher for the DPAGGR2 model than the DP-AGGR1 model both when using Price 1 and Price 2 distributions $(0.54$ vs. 0.36 and 0.61 and $0.53)$.

Table II further indicates that the underestimated charging costs of aggregate battery models are largely corrected when they are distributed to individual vehicles (DISTR1 and DISTR2 cases; less than $2 \%$ deviation in cost). This implies that the aggregate model-based approach can effectively be used on the aggregator level to provide charging reference for the distributed charging algorithm.

When the more time-variant Price model 2 is used, the trends in results are similar (cf. the four columns of Table II). In this case the DP-AGGR2 charging cost is overestimated by about $3 \%$ which is still found preferable when compared to the $7 \%$ cost underestimate when using the DP-AGGR1 approach. After distributing the optimized aggregate power to individual vehicles, the charging costs lie within the error margin of less than $0.5 \%$ error compared to the DP-IND approach.

The 2nd row of Table II includes the charging costs and the corresponding correlation coefficients for the case of omitting the $S o C$ constraint of being equal to one when a vehicle disconnects from the grid (see (14)). In this case, due to more optimization flexibility, the charging cost reduction potential is around $10 \%$ for the Price 1 model and $4 \%$ for the Price 2 model. A similar, but less emphasized trend is observed in the case of omitting the constraint on the grid power upper limit (3rd row of Table II).

TABLE II. ELECTRIC VEHICLE FLEET CHARGING COSTS COMPARISON FOR DIFFERENT OPTIMIZATION APPROACHES, TWO ELECTRICTY PRICE MODELS AND $20 \mathrm{KW}$ OF THE GRID POWER UPPER LIMIT

\begin{tabular}{|c|c|c|c|c|}
\hline Method & $\boldsymbol{C}_{\text {bat } 1}$ [EUR] & $\boldsymbol{K}_{\mathbf{1}}{ }^{\boldsymbol{}}[-]$ & $\boldsymbol{C}_{\text {bat } 2}$ [EUR] & $\boldsymbol{K}_{\mathbf{2}}{ }^{\boldsymbol{}}[-]$ \\
\hline DP-IND $^{\mathrm{a}}$ & $1258.3(-9.5 \%)$ & 0.81 & $829.6(-3.9 \%)$ & 0.76 \\
\hline DP-IND $^{\mathrm{c}}$ & $1385.5(-0.3 \%)$ & 0.92 & $858.6(-0.6 \%)$ & 0.70 \\
\hline DP-IND & $1390.1(0.0 \%)$ & 1.00 & $863.6(0.0 \%)$ & 1.00 \\
\hline DP-AGGR1 & $1099.7(-20.1 \%)$ & 0.36 & $805.7(-6.7 \%)$ & 0.53 \\
\hline DISTR1 & $1406.8(+1.2 \%)$ & 0.78 & $861.1(-0.3 \%)$ & 0.73 \\
\hline DP-AGGR2 & $1335.4(-3.9 \%)$ & 0.54 & $888.0(+2.8 \%)$ & 0.61 \\
\hline DISTR2 & $1416.2(+1.9 \%)$ & 0.80 & $865.4(+0.2 \%)$ & 0.82 \\
\hline
\end{tabular}

a. In this case SoC-related constraint (14) is omitted.

${ }^{\text {b. }} K_{1,2}$ - correlation between charging power obtained with DP-IND ( $4^{\text {th }}$ column $)$ and power profile obtained with another methods listed in Table II.

c. In this case upper limit on the grid power (16) is omitted.

Fig. 8 shows the cumulative upper limit of individual vehicle charging power for different vehicle optimizations within the successive optimization procedure. These results illustrate how the upper constraint of charging power is being reduced based on the iterative process (22) starting from the $1^{\text {st }}$ vehicle optimization, when it is at the maximum value, towards the last vehicle optimization, where this constraint is sometimes violated $\left(P_{\text {cum }, \text { lim }}<0\right)$. This can occur because this constraint is implemented as a soft constraint (16) and can be overruled by other constraints which have a higher priority.

Table III quantifies the likelihood values of the maximum grid power violation for the same optimization approaches considered in the Table II. The number of discrete time steps $N$ (hours) in which the maximum grid power is violated, the mean value of charging power $P_{c, \text { cum,mean }}$ in hours when this violation occurs, and the maximum charging power $P_{c, \text { cum }, \max }$ are taken as violation indices. The results indicate that all

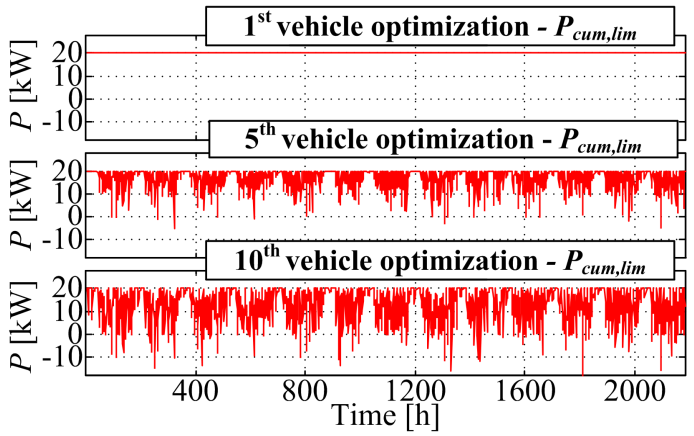

Fig. 8. Cumulative upper limit of maximum charging power from the grid $\left(P_{c \text { max }, \text { grid }}=20 \mathrm{~kW}\right)$. 
optimization approaches, except DP-AGGR1, violate the grid power constraint. However, this violation is significantly reduced (e.g. $N$ is by $52 \%$ lower) when the upper constraint on the grid power is included in the DP-IND optimization, and the constrained DP-IND optimization is less sensitive to the violation when compared to other two distributed charging methods DISTR1 and DISTR2.

TABLE III. QUANTIFICATION OF MAXIMUM GRID POWER VIOLATION AND COMPARISON FOR THE CASE OF DIFFERENT OPTIMIZATION APPROACHES, PRICE 1 MODEL AND THE GRID POWER UPPER LIMIT OF $20 \mathrm{KW}$

\begin{tabular}{|c|c|c|c|}
\hline Method & $\boldsymbol{N}[-]$ & $\begin{array}{c}\boldsymbol{P}_{c, \text { cum }, \text { mean }} \\
\left(\boldsymbol{P}_{c, \text { cum }} \boldsymbol{P}_{\text {cmax }, \text { grid }}\right)[\mathbf{k W}]\end{array}$ & $\boldsymbol{P}_{c, \text { cum }, \text { max }}[\mathbf{k W}]$ \\
\hline DP-IND $^{\mathbf{d}}$ & $186(0.0 \%)$ & $28.5(0.0 \%)$ & $58.6(0.0 \%)$ \\
\hline DP-IND & $89(-52.2 \%)$ & $25.6(-10.2 \%)$ & $46.8(-20.1 \%)$ \\
\hline DP-AGGR1 & $0(-100.0 \%)$ & N/A & $20.0(-65.9 \%)$ \\
\hline DISTR 1 & $156(-16.1 \%)$ & $25.0(-12.3 \%)$ & $49.9(-14.9 \%)$ \\
\hline DP-AGGR2 & $26(-86.0 \%)$ & $39.1(+37.2 \%)$ & $80.0(+36.5 \%)$ \\
\hline DISTR2 & $129(-30.7 \%)$ & $26.2(-8.1 \%)$ & $55.5(-5.3 \%)$ \\
\hline
\end{tabular}

${ }^{\text {d. }}$ In this case upper limit on the grid power (16) is omitted.

\section{CONCLUSIONS}

The paper has dealt with modelling and charging optimization of electric vehicle fleet. The distributed electric vehicle fleet model is obtained through battery modelling of each electric vehicle within the fleet. The battery model of each vehicle is parameterized by using the recorded data related to delivery vehicle fleet of a retail company. Charging optimization is performed by using the dynamic programming algorithm. Each vehicle charging schedule is optimized separately while taking into account common maximum power which can be drawn from the grid.

The optimization results show that the optimization ordering of vehicles charging does not have a large influence on the final fleet charging cost. Since this individual vehicle modelling approach captures realistic constraints, which is not fully the case with aggregate battery modelling approach, the obtained results are used to validate the previously developed aggregate battery models. The charging optimization of the novel aggregate battery model is shown to be more realistic than the existing (basic) aggregate battery model, so that the novel model can be conveniently used for energy planning or smart charging studies which involve a large number of electric vehicles.

\section{ACKNOWLEDGMENT}

It is gratefully acknowledged that this work has been supported by the Croatian Science Foundation through the project No. 09/128. The data and technical support from Konzum d.d. is greatly appreciated, as well.

\section{REFERENCES}

[1] B. A. Davis, M. A. Figliozzi, "A methodology to evaluate the competitiveness of electric delivery trucks", Transportation Research Part E, Vol. 49, Issue 1, pp 8-23, 2013.

[2] B. Škugor, J. Deur, "Delivery Vehicle Fleet Data Collection, Analysis, and Naturalistic Driving Cycles Synthesis", International Journal of Innovation and Sustainable Development (IJISD), 2014. (submitted)

[3] N. Rotering, M. Ilic, "Optimal Charge Control of Plug-In Hybrid Electric Vehicles in Deregulated Electricity Markets", IEEE Transactions on Power Systems, Vol. 26, No. 3, pp. 1021-1029., 2011.

[4] S. Han, H. H. Soo, K. Sezaki, "Design of an optimal aggregator for vehicle-to-grid regulation service", Innovative Smart Grid Technologies Conference (ISGT), art. no. 5434773., 2010.

[5] O. Sundström, C. Binding, "Flexible Charging Optimization for Electric Vehicles Considering Distribution Grid Constraints", IEEE Transaction on Smart Grid, 2012.

[6] T. Lan, J. Hu, Q. Kang, C. Si, L. Wang, Q. Wu, "Optimal control of an electric vehicle's charging schedule under electricity markets", Neural Computing and Applications, vol. 23, No. 7-8, pp. 1865-1872., 2013.

[7] H. Lund, W. Kempton, "Integration of renewable energy into the transport and electricity sectors through V2G", Energy Policy, Vol. 36 , pp. 3578-3587, 2008.

[8] J. Xu, V.W.S. Wong, "An approximate dynamic programming approach for coordinated charging control at vehicle-to-grid aggregator", IEEE International Conference on Smart Grid Communications, art. no. 6102333, pp. 279-284., 2011.

[9] B. Škugor, J. Deur, "Dynamic Programming-based Optimisation of Charging an Electric Vehicle Fleet System Represented by an Aggregate Battery Model", $9^{\text {th }}$ Conference on Sustainable Development of Energy, Water and Environment Systems (SDEWES), Dubrovnik, Croatia, 2014.

[10] B. Škugor, J. Deur, “A Novel Model of Electric Vehicle Fleet Aggregate Battery for Energy Planning Studies", $9^{\text {th }}$ Conference on Sustainable Development of Energy, Water and Environment Systems (SDEWES), Dubrovnik, Croatia, 2014.

[11] M. A. Miller, A. G. Holmes, B. M. Conlon and P. J. Savagian, "The GM "Voltec" 4ET50 Multi-Mode Electric Transaxle", SAE Paper 2011-010887, 2011.

[12] M. Cipek, B. Škugor and J. Deur, "Comparative Characteristics of Conventional and Electric Delivery Vehicles Based on Realistic Driving Cycles", European Electric Vehicle Congress (EEVC 2014), Brussels, Belgium, 2014. (accepted)

[13] J. Deur, M. Cipek, B. Škugor, and J. Petrić, "Modeling and Low-level Control of Range Extended Electric Vehicle Dynamics," International Conference on Powertrain Modeling and Control (PMC 2012), Bradford, UK, 2012.

[14] Škugor, B., Deur, J., "Instantaneous Optimization-based Energy Management Control Strategy for Range Extended Electric Vehicle", SAE Technical Paper 2013-01-1460, 2013.

[15] R. E. Bellman, and S. E. Dreyfus, "Applied Dynamic Programming", Princeton University Press, Princeton, New Jersey, USA, ISBN 978-0691-07913-4,1962.

\section{APPENDIX: DP OPTIMIZATION PARAMETERS}

$P_{c \text { max }, \text { grid }}=20 \mathrm{~kW}, P_{c, \text { min }}=0 \mathrm{~kW}, P_{c, \text { lim }}=10 \mathrm{~kW}, S o C_{\text {low, lim }}=0, S o C_{\text {upp,lim }}=1$, $N_{\text {control }}=1000, N_{\text {soc }}=100, N_{v}=10, K_{f}=10^{10}, K_{g 1}=K_{g 2}=K_{g 3}=10^{8}, K_{g 4}=10^{5}$, $K_{g 5}=K_{g 7}=K_{g 8}=K_{g 9}=10^{3}, K_{g 6}=10^{4}, \Delta T=1 \mathrm{~h}, N_{t}=2184 \mathrm{~h}$ (for $t_{f}=3$ months and $\Delta T=1 \mathrm{~h}), E_{\max }=16 \mathrm{kWh}, E_{\max , a g g}=160 \mathrm{kWh}, \eta_{c h}=\eta_{\text {dch }}=0.92, S o C_{\text {init }}=$ 